\title{
Deep Belief Networks for Quantitative Analysis of A Gold Immunochromatographic Strip
}

\author{
Nianyin Zeng, Zidong Wang*, Hong Zhang, Weibo Liu and Fuad E. Alsaadi
}

\begin{abstract}
Gold immunochromatographic strip (GICS) has become a popular membrane-based diagnostic tool in a variety of settings due to its sensitivity, simplicity and rapidness. This paper aims to develop a framework of automatic image inspection to further improve the sensitivity as well as the quantitative performance of the GICS systems. As one of the latest methodologies in machine learning, the deep belief network (DBN) is applied, for the first time, to quantitative analysis of GICS images with hope to segment the test and control lines with a high accuracy. It is remarkable that the exploited DBN is capable of simultaneously learning three proposed features including intensity, distance and difference to distinguish the test and control lines from the region of interest that are obtained by pre-processing the GICS images. Several indices are proposed to evaluate the proposed method. The experiment results show the feasibility and effectiveness of the DBN in the sense that it provides a robust image processing methodology for quantitative analysis of GICS.
\end{abstract}

\section{Index Terms}

Gold immunochromatographic strip; deep belief networks (DBNs); restricted Boltzmann machine (RBM); quantitative analysis; image segmentation.

\section{INTRODUCTION}

Deep belief network (DBN), proposed by Hinton [8] in 2006, is an extensively studied and widely used deep learning model. It is remarkable that the deep learning model is a biologically inspired model which mimics the layered structure of the cortex [30]. Essentially, DBN is a greedy and multi-layer formed learning model combined by a stack of restricted Boltzmann machines (RBMs). Unlike other multi-layer and nonlinear models, the distinct merit of DBN is its capability of obtaining the states of hidden layers units by one forward pass. In the last few years, DBN has drawn increasing research attention in many application fields such as recognition, signal and information processing, image processing and classification [6], [9], [19], [20], [26], [30], [33]. Therefore, we propose to use the DBN approach for quantitative analysis of a gold immunochromatographic strip.

Gold immunochromatographic strip (GICS), labeled with the colloidal gold nanoparticle, is on the basis of an immunochromatographic process that utilizes the high specificity of antigen-antibody reaction and provides rapid determination of target analyte. In the past decade, the GICS has been extensively studied

This work was supported in part by the Natural Science Foundation of China under Grant 61403319, in part by the Fujian Natural Science Foundation under Grant 2015J05131, in part by the Fujian Provincial Key Laboratory of Eco-Industrial Green Technology, and in part by the Fundamental Research Funds for the Central Universities.

N. Zeng and H. Zhang are with the Department of Mechanical and Electrical Engineering, Xiamen University, Fujian 361005, China.

Z. Wang is with the Department of Computer Science, Brunel University London, Uxbridge, Middlesex, UB8 3PH, United Kingdom. He is also with the Communication Systems and Networks (CSN) Research Group, Faculty of Engineering, King Abdulaziz University, Jeddah 21589, Saudi Arabia. Email address: Zidong.Wang@brunel.ac.uk

W. Liu is with the Department of Computer Science, Brunel University London, Uxbridge, Middlesex, UB8 3PH, United Kingdom.

F. E. Alsaadi is with the Communication Systems and Networks (CSN) Research Group, Faculty of Engineering, King Abdulaziz University, Jeddah 21589, Saudi Arabia.

${ }^{*}$ Corresponding author. 
and widely applied to the biomedical and related areas for determination of analytes in specimens due to its fascinating advantages including short assay time, ease of use, good specificity and satisfactory stability [21], [24], [32]. Up to now, a variety of material-selection-based approaches have been introduced by biochemical researchers to enhance the quantification performance of GICS, see e.g. [12], [14], [27]). On the other hand, it should be highlighted that the research on modeling the biochemical reactions of GICS with aim to optimize assay performance has stirred considerable research interest, see e.g. [22], [23], [37]-[39], [41], [42], [44]. A focus of research in this area has been on how to exploit the quantitative instruments of GICS for more sensitive and quantitative performance, see [1], [5], [11], [13], [15], [16], [25], [40], [43] and the references therein.

Among many available detection schemes for quantitative instruments developed throughout the years, the most frequently used methods rely on the reflectance photometers to obtain the GICS signals, see e.g. [5], [11], [16]. At the same time, there has been a growing research interest in the development of image-based instruments for GICS, see e.g. [1], [13], [15], [25], [40], [43]. The critical design specification for image-based system is the image processing technology whose significance has now been well recognized in the bioinformatics community. In particular, it is of vital importance to look for an efficient image segmentation method to accurately distinguish the test and control lines from the GICS image. In recent years, a number of methods have been introduced to achieve the goal of segmenting the test and control lines of GICS images. Some typical methods include the Otsu threshold segmentation approach, the fuzzy c-means (FCM) clustering method and the cellular neural network (CNN) [1], [15], [25], [40], [43]. A common limitation yet a major challenge for these methods, however, is that the acquired GICS image usually involves unavoidable noises caused by various factors including temperature, humidity, colloidal gold and non-uniform permeation of specimens. Also, it is often difficult to ensure the accuracy for images of low concentration where the noises take a great proportion and the signal-noise-ratio is therefore small (i.e. the intensities of some background noises are much higher than the signals) [43]. To address these issues, we propose to use the recently developed deep belief network (DBN) algorithm, a state-of-the-art machine learning technique, for the GICS image segmentation in this paper.

It should be mentioned that it is a challenging task to process the GICS images due to their inherent features outlined as follows. First, in order to enhance the efficiency, we just concentrate on the region of interest with the test and control lines immobilized on the strip. Therefore, the obtained GICS images should be pre-processed to acquire the region of interest via some commonly used image segmentation operators. Second, both lines in the reading window might become blurred, uncertain and mixed with the background since they are generally made/smeared via a roller in a non-uniform manner. In addition, when the sample to be detected (such as urine, blood, serum) flows over the membrane, some interference noises are inevitable on the detection window of strip [43]. In order to overcome the challenges mentioned above, we intend to establish a DBN-based framework for quantitative analysis of the GICS by accurately extracting the test and control lines from the acquired GICS images.

The main novelty of our work is primarily twofold. 1) A new framework of automatic image inspection is established to solve the problems in the quantitative evaluation of gold immunochromatographic strip, where the DBN algorithm is applied to accurately extract the test and control lines. 2) By learning three features including intensity, distance and difference, the presented DBN can distinguish the test and control lines from the region of interest that is obtained by pre-processing the GICS images. It is shown from the experiment results that the proposed method provides high accuracy in terms of the performance of the segmentation, the feature parameter, the fitting line and the peak signal-to-noise ratio.

The remainder of this paper is organized as follows. In Section II, the gold immunochromatographic 
strip assay and the problem formulation are presented. Section III provides a detailed introduction on the restricted Boltzmann machine, the deep belief networks, as well as the applications in the segmentation of GICS images. Section IV mainly discusses the performance of image segmentation via the deep belief network and also evaluates its overall performance in terms of some well-defined criteria. Finally, conclusions are drawn in Section V.

\section{The Gold Immunochromatographic Strip Image And Problem Formulation}

Gold immunochromatographic strip, which is labeled with the colloidal gold nanoparticle, is on the basis of an immunochromatographic process that utilizes the high specificity of antigen-antibody reaction and provides rapid determination of target analyte. The GICS, as shown in Fig. 1, is formed by a variety of constituents including a sample pad, a conjugate pad, an absorbent pad, and a nitrocellulose membrane on which the reaction occurs. There are generally two formats of GICS, namely, sandwich and competitive formats. Here, we only discuss the sandwich format which uses two antibodies to bind the analyte in between. With the presence of an antigen in the sample, a sandwich-type compound is formed between the labeled antibody and the antibody immobilized on the membrane. After that, the red or purple red color caused by the accumulation of gold nanoparticle at the test and the control lines would appear on the membrane. Particularly, the signal intensity of the test line is directly related to the concentration of the target analyte in the samples. Therefore, the concentration of the target analyte can be assessed visually or by a reader system for quantitative analysis by monitoring the signal of the sandwich-type compound on the test line [37]-[39].

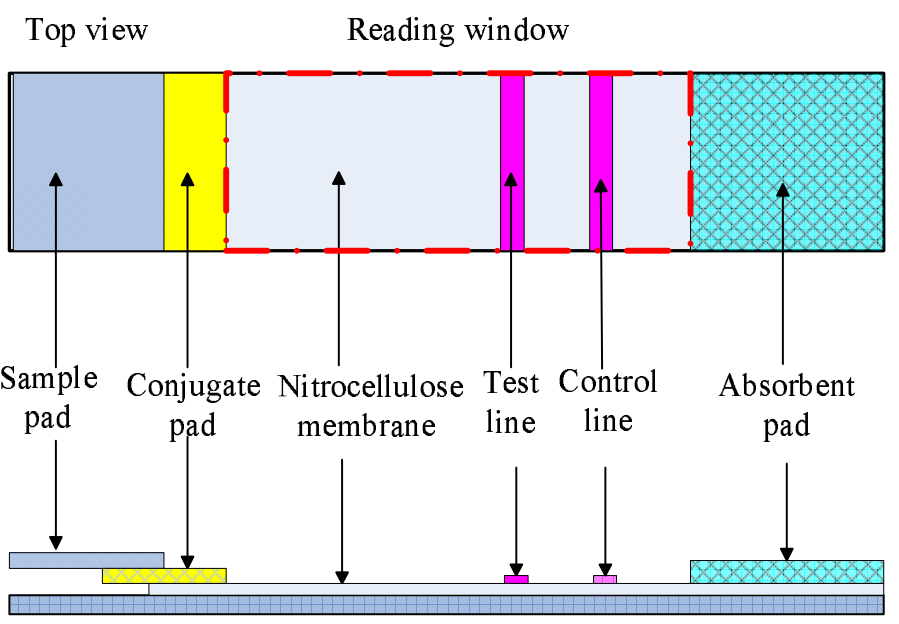

Cross section

Fig. 1. The schematic structure of the gold immunochromatographic strip

In this paper, the human chorionic gonadotropin (hCG) is selected as the target analyte in experiments. It is of great significance to quantitatively determine the concentration of hCG that can be used as indicators of a number of diseases such as ectopic pregnancy differentiation and fetal Down syndrome screening. In particular, ten different concentrations of hCG have been added into GICS strips in the experiments and, therefore, we can capture GICS images at the fixed time points as shown in Fig. 2.

Remark 1: As discussed in the introduction, there are essential difficulties in processing the GICS image because of 1) an additional step of pre-processing introduced to acquire the region of interest through commonly used image segmentation operators; 2) the blurriness of the test and control lines resulting from their production process; and 3) the interference noises existing on the detection window of strip. 
It is, therefore, the main objective of this paper to overcome the difficulties identified above by launching a quantitative analysis on the GICS via accurately segmenting the test and control lines from the acquired GICS images.

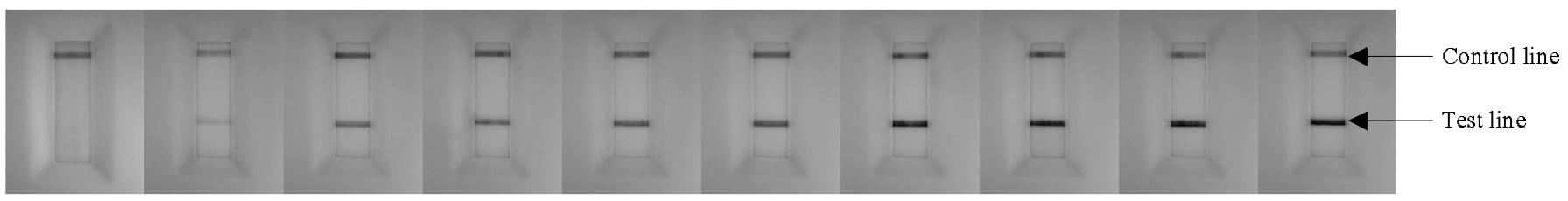

Fig. 2. Images of GICS with different concentrations(from the left side: $0,10,35,75,100,150,200,300,400,500 \mathrm{mIU} / \mathrm{ml}$ ).

\section{DeEP Belief Networks For QuAntitative AnAlysis of The GICS}

In this section, we introduce the restricted Boltzmann machine and the deep belief networks (see e.g. [8], [9], [20], [26], [33] for more details), which will be applied to the GICS image segmentation when learning features to distinguish the test and control lines.

\section{A. Restricted Boltzmann Machine}

The restricted Boltzmann machine (RBM) [8], as shown in Fig. 3, is a bipartite graph in which visible units $v$ are linked to hidden units $h$ through undirected weighted connections. In general, visible units represent observations, and hidden units tend to represent features. A special characteristic of RBM is that there are no connections in any two visible units and also any two hidden units. Due to different situations, there are two types of RBMs, namely, Bernoulli-Bernoulli RBM with binary visible and hidden units, and Gaussian-Bernoulli RBM where hidden units are binary but the visible units are linear with Gaussian noise.

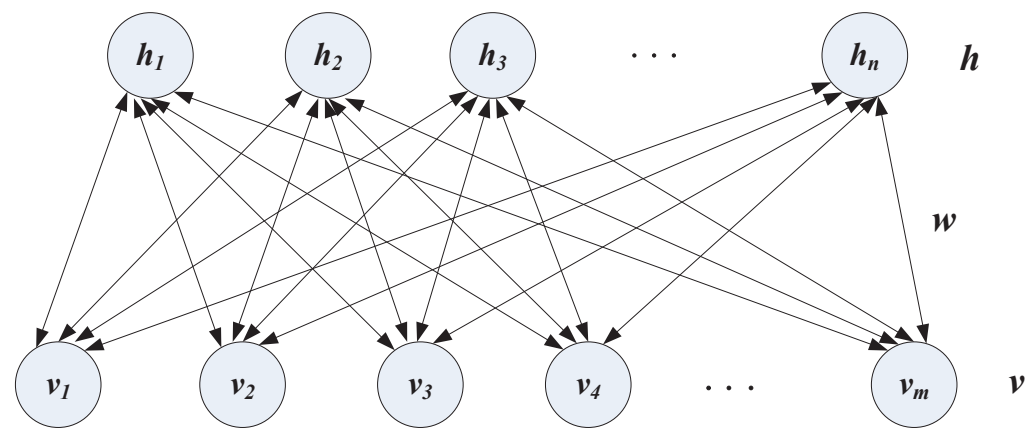

Fig. 3. Schematic diagram of RBM.

In an RBM, the joint probability distribution of visible and hidden units $p(v, h \mid \theta)$ is defined by the energy function. For a given state $(v, h)$, the energy function of Bernoulli-Bernoulli RBM is [8]:

$$
E(v, h \mid \theta)=-\sum_{i=1}^{m} \sum_{j=1}^{n} w_{i j} v_{i} h_{j}-\sum_{i=1}^{m} b_{i} v_{i}-\sum_{j=1}^{n} a_{j} h_{j}
$$

where $\theta=(w, b, a)$ denotes the unknown model parameter, $w_{i j}$ is the connection weight between $v_{i}$ and $h_{j}$, and $b_{i}$ and $a_{j}$ are their biases, respectively. $m$ and $n$ represent the numbers of visible and hidden units. 
As such, based on the energy function, the joint probability distribution $p(v, h \mid \theta)$ is given as follows:

$$
p(v, h \mid \theta)=\frac{e^{-E(v, h \mid \theta)}}{Z(\theta)}
$$

where $Z(\theta)=\sum_{v, h} e^{-E(v, h \mid \theta)}$ represents the normalization factor or the partition function. Then, the marginal probability with which the model assigns to a visible vector $v$ (also called likelihood function) is given by:

$$
p(v \mid \theta)=\frac{\sum_{h} e^{-E(v, h \mid \theta)}}{Z(\theta)}
$$

Because there are no connections in any two visible units and also any two hidden units, the conditional probabilities $p(h \mid v, \theta)$ and $p(v \mid h, \theta)$ are factorial and can be calculated by:

$$
\begin{aligned}
& p\left(h_{j}=1 \mid v, \theta\right)=\sigma\left(\sum_{i=1}^{m} w_{i j} v_{i}+a_{j}\right) \\
& p\left(v_{i}=1 \mid h, \theta\right)=\sigma\left(\sum_{j=1}^{n} w_{i j} h_{j}+b_{i}\right)
\end{aligned}
$$

where $\sigma(x)=\left(1+e^{-x}\right)^{-1}$.

The purpose of iteration in $\mathrm{RBM}$ is to find an appropriate parameter $\theta=(w, b, a)$ so as to fit the given training data. Actually, $\theta=(w, b, a)$ can be computed via maximizing the log likelihood function in the given training data ( $T$ denotes the size of the training data):

$$
\theta^{*}=\underset{\theta}{\arg \max } \sum_{t=1}^{T} \ln p\left(v^{(t)} \mid \theta\right)
$$

According to the contrastive divergence (CD) algorithm proposed by Hinton [7], the update rules are given as follows:

$$
\begin{aligned}
& \Delta w_{i j}=\epsilon\left(\left\langle v_{i} h_{j}\right\rangle_{\text {data }}-\left\langle v_{i} h_{j}\right\rangle_{\text {recon }}\right) \\
& \Delta b_{i}=\epsilon\left(\left\langle v_{i}\right\rangle_{\text {data }}-\left\langle v_{i}\right\rangle_{\text {recon }}\right) \\
& \Delta a_{j}=\epsilon\left(\left\langle h_{j}\right\rangle_{\text {data }}-\left\langle h_{j}\right\rangle_{\text {recon }}\right)
\end{aligned}
$$

where $\epsilon$ is the learning rate, $\langle\cdot\rangle_{\text {data }}$ means the expectation of distribution defined by the training data, and $\langle\cdot\rangle_{\text {recon }}$ represents the expectation of distribution defined by the reconstruction model.

Similarly, the energy function of Gaussian-Bernoulli RBM is:

$$
E(v, h \mid \theta)=\sum_{i=1}^{m} \frac{\left(v_{i}-b_{i}\right)^{2}}{2}-\sum_{i=1}^{m} \sum_{j=1}^{n} w_{i j} v_{i} h_{j}-\sum_{j=1}^{n} a_{j} h_{j}
$$

and the corresponding conditional probabilities can be computed as follows:

$$
\begin{array}{r}
p\left(h_{j}=1 \mid v, \theta\right)=\sigma\left(\sum_{i=1}^{m} w_{i j} v_{i}+a_{j}\right) \\
p\left(v_{i} \mid h, \theta\right)=N\left(\sum_{j=1}^{n} w_{i j} h_{j}+b_{i}, 1\right)
\end{array}
$$

where $N(\mu, \delta)$ represents a Gaussian distribution with mean $\mu$ and variance $\delta$. 


\section{B. Deep Belief Network}

Deep Belief Network (DBN) was proposed by Hinton [8] in 2006 and, since then, DBN has been extensively investigated and widely employed in both theory and applications of various deep learning tasks. As shown in Fig. 4, the DBN is a neural network constructed from multi-layer RBM and one-layer Backpropagation (BP). DBN is actually a greedy and multi-layer-formed learning model combined with a stack of RBMs. A distinguishing feature of the DBN is its capability of obtaining states of hidden layers units by one forward pass. In the course of training a DBN, the first step is the so-called pre-training that stacks a lot of RBMs layer by layer in a bottom-up manner. Once the parameters of the lower-layer RBM are determined by learning, the vectors of hidden feature activations can be utilized as the input of visible units for the higher-layer RBM. Then, in the fine-tuning stage, the error back propagation approach is utilized to adjust the weights of whole network.

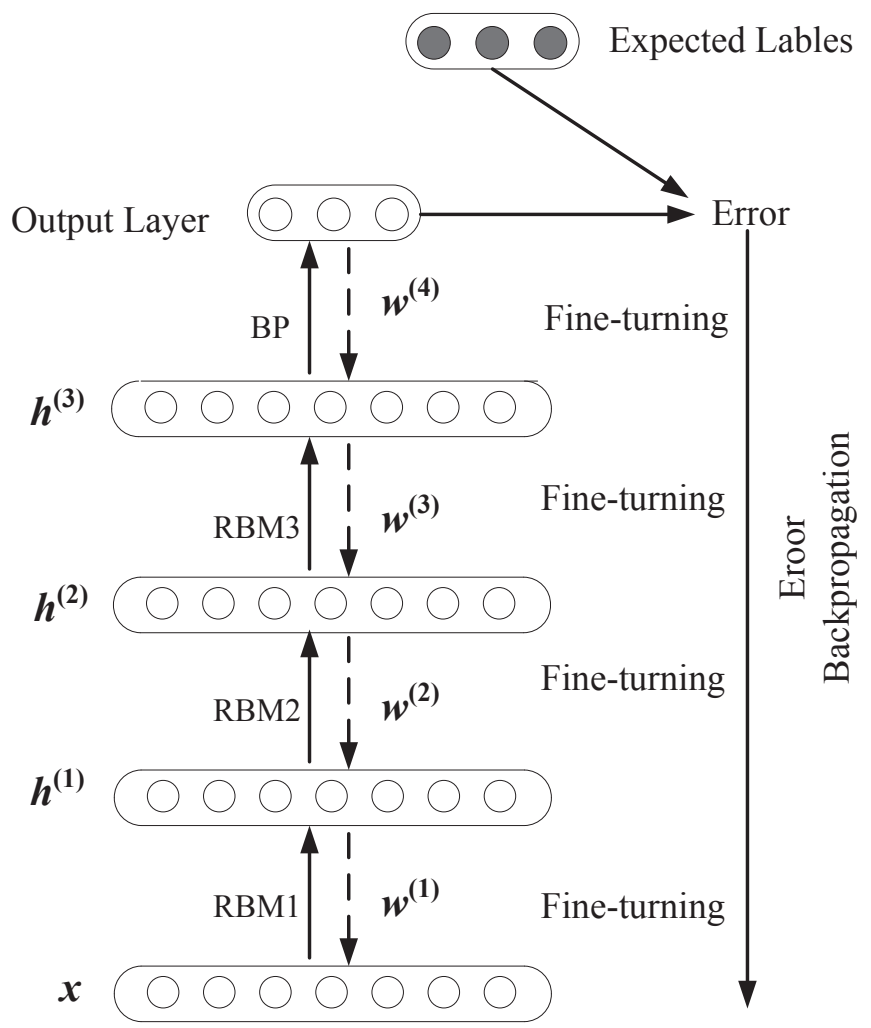

Fig. 4. Schematic diagram of a DBN.

For the $i$ th node of the output layer, we suppose that the actual output is $o_{i}$ and the expected output is $d_{i}$. The sensitivity $\delta_{i}$ can be calculated by:

$$
\delta_{i}=o_{i}\left(1-o_{i}\right)\left(d_{i}-o_{i}\right)
$$

For the $l$ th hidden layer, letting $y_{i}$ be the output of $i$ th node, the sensitivity $\delta_{i}$ can be conducted according to

$$
\delta_{i}^{l}=y_{i}^{l}\left(1-y_{i}^{l}\right) \sum_{j} w_{i j}^{l} j_{j}^{l+1}
$$

and, finally, the weights of DBN are updated by:

$$
\begin{aligned}
& w_{i j}^{l}=w_{i j}^{l}+\varepsilon_{\text {fine-tuning }} y_{j}^{l} \delta_{j}^{l+1} \\
& b_{j}^{l}=b_{j}^{l}+\varepsilon_{\text {fine-tuning }} \delta_{j}^{l+1}
\end{aligned}
$$




\section{Process of GICS Image via Deep Belief Networks}

In this section, we introduce the process of GICS image via the deep belief network. The flowchart of the DBN-based GICS image segmentation is shown in Fig. 5 and our aim is to learn features to distinguish the test and control lines from the acquired strip images.

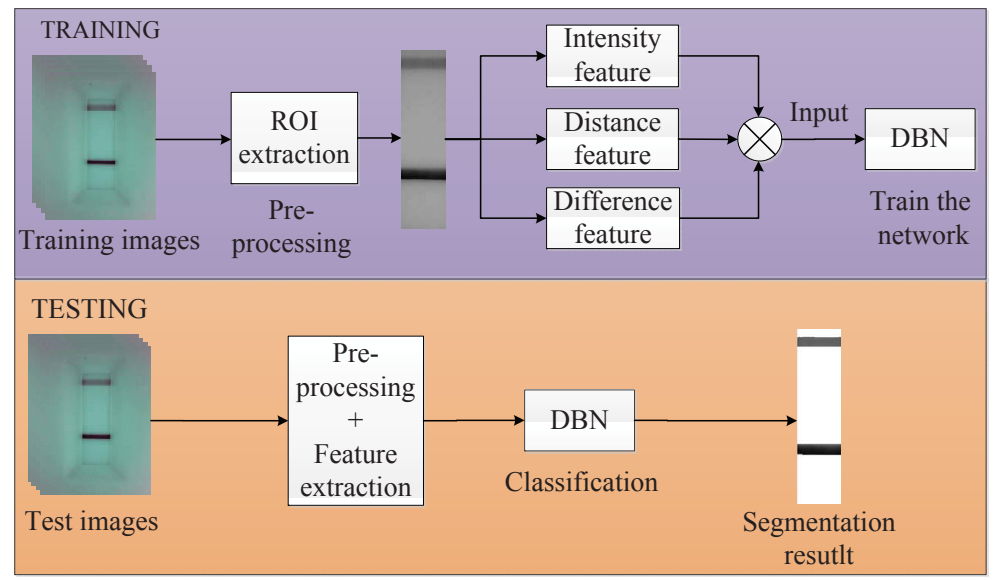

Fig. 5. Flowchart of the DBN-based GICS image segmentation.

The main objective of segmenting the GICS image is to determine whether pixels in the image belong to the test line or the control line and, therefore, it can be regarded as a classification problem. In general, the size and pixel numbers of the images acquired from strip are slightly different, and this is not conducive to the image processing. Therefore, the acquired images should be pre-processed at first so as to extract region of interest (ROI) with the fixed size. The selection of the input feature is particularly important for the DBN as it plays a key role in the performance of classification. In this paper, each pixel in the image is treated as a sample, and three factors have been taken into account for selecting the input feature of DBN.

First of all, the gray intensity of pixels in the neighborhood should be considered since the intensity of test and control lines are generally larger than the surrounding areas. If the square window size is set as winsize, a vector with size winsize * winsize can be obtained. For pixels near the image border, we use the mirroring method [26] to obtain intensity values of regions inside the window but beyond the image border. However, as is well known, with the analyte concentration increasing, the color in test and control lines will deepen, while the corresponding intensity values of pixels will decrease. Based on this observation, we introduce two input features, one is the distance feature that represents the distance to the center, and the other is the difference feature that represents the difference of intensity values between two lines and the background. By doing so, the DBN approach can perform well even when analyte concentration is at a low level. It should be noted here that all input features of DBN should be normalized.

In the training stage, three features are utilized as the input of the visible layer of the first RBM. After training layer by layer, the correlation of input data in time and space is mapped to hidden layers successively. Particularly, the batch method is exploited to update the weights so as to speed up the training rate. In the prediction process, the classification results can be calculated by forward propagation of the trained DBN. 


\section{EXPERIMENTAL RESULTS AND DisCUSSIONS}

\section{A. DBN for GICS Image Segmentation Results}

Considering the distinguished features of the GICS images, we divide the extracted ROI into two parts for segmenting in order to reduce the complexity of algorithm and avoid unnecessary calculations. One part consists of the control line and corresponding background, while the other part consists of the test line and its background. Based on the DBN approach mentioned above, all parameters of the DBN have been fixed for all experiments. Firstly, we find that the performance of segmentation is best when the network has two RBM layers and each layer has 100 hidden nodes. Next, we set the learning rate as 1 in the pre-training stage as well as the fine-tuning state, and the mini-batch sizes for both stages are set as 100 and 50 respectively. Finally, the number of iteration is set as 20 because the classification accuracy tends to stable after that.

As for the training sample, we use 18 images with different level of analyte concentrations (from low to high). The window size winsize is 13 and the dimension of input data is thus 171 . The extracted region of interest (ROI) is selected as $50 * 90$ in each image and, accordingly, the number of training sample is $18 * 50 * 90$.

For the purpose of showing the performance of segmentation comprehensively, we choose different level of analyte concentrations as testing sample. Three typical simulation results by using DBN approach for segmenting the GICS images are shown in Fig. 6 when the concentrations of the target analyte are, respectively, $75 \mathrm{ml}, 200 \mathrm{ml}$ and $500 \mathrm{ml}$. Furthermore, the classification accuracy of all testing images are listed in Table. I

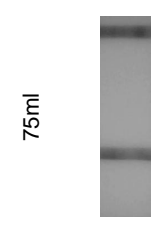

(a)

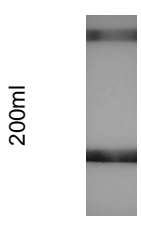

(c)

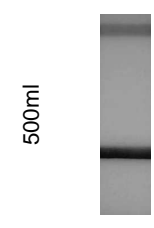

(e)

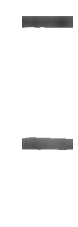

(b)

(d)

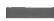

(f)

Fig. 6. Three typical simulation results of DBN approach for segmenting the GICS images. Left column: Simulation results of extracted ROI; Right column: Simulation results of DBN approach for segmenting the GICS images.

From Fig. 6, we can see that the DBN approach provides a robust method for accurately extracting both lines from the GICS images with different concentrations of the target analyte. Especially, the concentration of specimens in the GICS images can be low, middle or high. It can also be verified from Table. I that the 
TABLE I

THE CLASSIFICATION ACCURACY OF ALL TESTING IMAGES

\begin{tabular}{|c|c|c|c|}
\hline \hline Concentration & Control line error ratio (\%) & Test line error ratio (\%) & Total accuracy ratio (\%) \\
\hline $35 \mathrm{ml}$ & 3.18 & 3.69 & 96.565 \\
\hline $75 \mathrm{ml}$ & 0.56 & 2.00 & 98.720 \\
\hline $100 \mathrm{ml}$ & 0.29 & 4.80 & 97.455 \\
\hline $150 \mathrm{ml}$ & 0.76 & 1.73 & 98.755 \\
\hline $200 \mathrm{ml}$ & 3.47 & 1.93 & 97.300 \\
\hline $300 \mathrm{ml}$ & 1.62 & 4.96 & 96.710 \\
\hline $400 \mathrm{ml}$ & 2.00 & 2.62 & 97.690 \\
\hline $500 \mathrm{ml}$ & 1.22 & 0.27 & 99.255 \\
\hline Average & 1.64 & 2.75 & 97.805 \\
\hline
\end{tabular}

DBN method in this paper has a satisfactory performance in image segmentation with high classification accuracy. Therefore, the DBN has proven to be a novel approach for quantitative analysis of GICS systems.

\section{B. Feature Calculation and Line Fitting}

For the purpose of quantification, a feature parameter should be exploited to interpret the concentration of the specimens. According to the Lambert-Beer law, a feature parameter named relative integral optical density $(R I O D)[15]$ is presented to evaluate the concentration of target analyte. The RIOD is given as follows:

$$
R I O D=\frac{I O D_{T}}{I O D_{C}}=\frac{\sum_{i=1}^{N} \lg \frac{G_{0}}{G_{T}}}{\sum_{j=1}^{M} \lg \frac{G_{0}}{G_{C}{ }^{j}}}
$$

where $I O D$ is the abbreviation for integral optical density, and $I O D_{T}$ and $I O D_{C}$ denote the $I O D$ of the test and control lines, respectively. $G_{T}$ and $G_{C}$ describe the gray intensity of pixels on the test and control lines, respectively. $G_{0}$ represents the mean gray intensity of the reading window area.

As shown in Fig. 7, a straight line is fitted for describing the relationship between the concentration and the $R I O D$ via the least square approach. Especially, the horizontal axis stands for the hCG concentrations, while the y-coordinate denotes the corresponding value of $R I O D$ which is calculated according to Eq. (17).

It is obvious that the RIOD and the $\mathrm{hCG}$ concentration have good corresponding relationships by the presented DBN approach and the adaptive cellular neural network $(\mathrm{CNN})$ [40] from the Fig. 7. The correlation coefficient of the presented DBN approach is 0.97681 , while the correlation coefficient of the adaptive $\mathrm{CNN}$ is 0.9689. Therefore, the presented DBN approach opens up a new way of image-based method for quantitative analysis of GICS system.

\section{Quantitative Evaluation}

In this part, a well-defined quality criterion called the peak signal-to-noise ratio (PSNR) [36] is exploited to quantify the segmentation performance of DBN method for GICS images, and also verify the effectiveness of the test and control lines in this study. In general, the PSNR is viewed as a measurement of peak error. In order to calculate the PSNR, the presented DBN approach firstly generates a binary mask which classifies the pixels of GICS image as two types, one is classified as the signal (test or control 


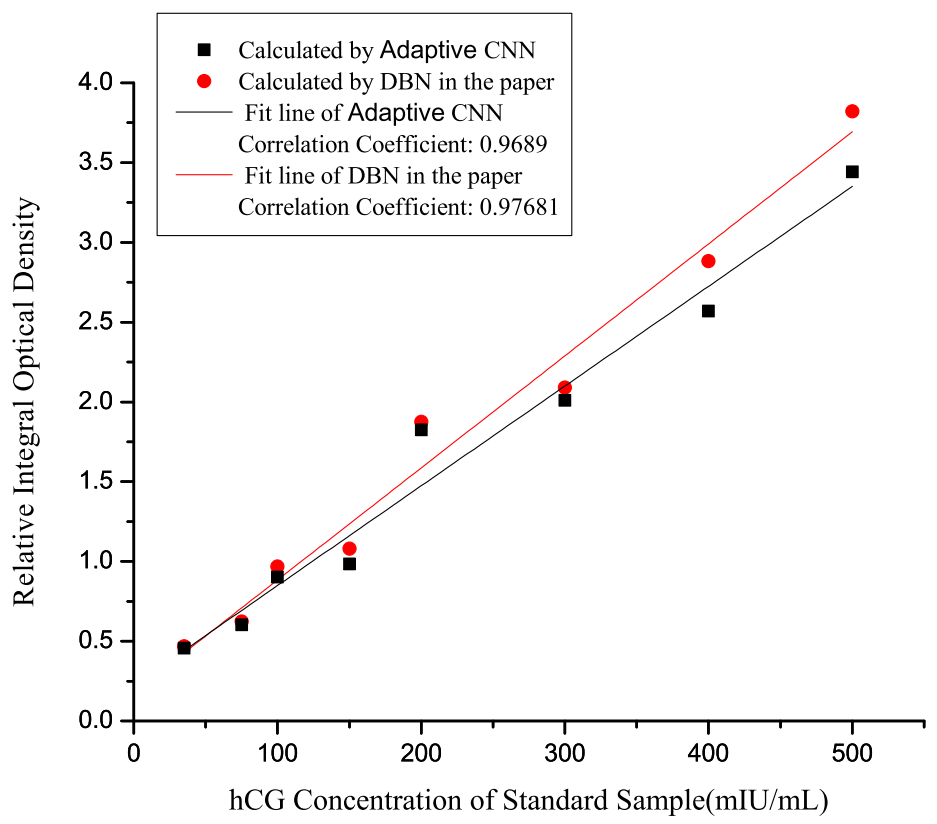

Fig. 7. The fitted line between the concentration hCG specimens and the RIOD via the least square approach.

line, assigned to 1) and the other belongs to the background (assigned to 0). After that, the PSNR can be calculated as follows:

$$
P S N R=10 \log \left[\frac{R^{2}}{M S E}\right]
$$

where $R$ stands for the maximum range of the image data type. For example, $R$ equals to 1 when the data type of input image is double-precision floating-point, and $R$ equals to 255 when the data type is an 8-bit unsigned integer, etc. MSE denotes the cumulative squared error between the binary mask $I_{1}$ and the normalized original image $I_{2}$, which can be obtained by:

$$
M S E=\frac{\sum_{M, N}\left[I_{1}(m, n)-I_{2}(m, n)\right]^{2}}{M \times N}
$$

where $M$ and $N$ represent the numbers of rows and columns in the input images, respectively.

As an important performance indicator, the PSNR describes the ratio of the signal's peak value over the magnitude of the background noise. Generally, we prefer a larger PSNR value since the binary spot mask in this way fits better with the raw image surface [36]. Fig. 8 shows the PSNR values of DBN method utilized for segmenting GICS images of 8 different concentrations of hCG. It is obvious that the DBN method possesses good performance of segmentation, which provides higher accuracy than the other existing methods for segmenting the GICS images shown in the [43].

\section{CONCLUSIONS}

In this paper, we have developed a novel approach to quantitative analysis of a gold immunochromatographic strip (GICS) using deep belief network (DBN) in order to enhance the robustness when accurately segmenting the test and control lines from the GICS images. Three features (including intensity, distance and difference) have been proposed for the DBN method to learn in order to successfully distinguish the test and control lines from the region of interest that is obtained by pre-processing the GICS images. 


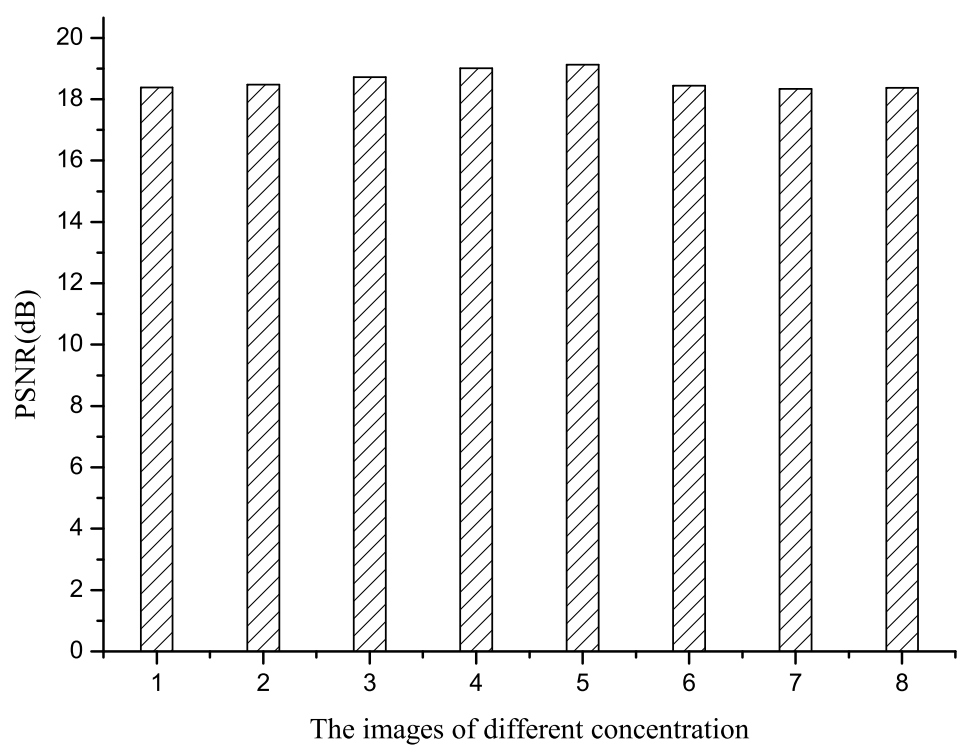

Fig. 8. The PSNR comparison of segmenting images.

Experiments have been carried out on different concentrations of hCG images. Furthermore, several indices have also been proposed to verify the presented DBN method and demonstrate that the DBN approach indeed gives high accuracy. Future research directions would include the modification of DBNs via adopting adaptively control strategies (e.g. [2]-[4], [10], [17], [18], [28], [29], [34], [35]) so as to further improve the performance of DBNs, and also developing more advanced image segmentation methods (e.g. [31], [45]) for quantitative analysis of a GICS.

\section{COMPliance With Ethical StAndards}

Conflict of Interest Nianyin Zeng, Zidong Wang, Hong Zhang, Weibo Liu and Fuad E. Alsaadi declare that they have no conflict of interest.

Informed Consent All procedures followed were in accordance with the ethical standards of the responsible committee on human experimentation (institutional and national) and with the Helsinki Declaration of 1975, as revised in 2008 (5). Additional informed consent was obtained from all patients for which identifying information is included in this article.

Human and Animal Rights This article does not contain any studies with human or animal subjects performed by the any of the authors.

\section{REFERENCES}

[1] L. Chuang, J. Hwang, H. Chang, F. Chang and SH. Jong, Rapid and simple quantitative measurement of $\alpha$-fetoprotein by combining immunochromatographic strip test and artificial neural network image analysis system, Clinica Chimica Acta., vol. 348, pp. 87-93, 2004.

[2] D. Ding, Z. Wang, B. Shen and G. Wei, Event-triggered consensus control for discrete-time stochastic multi-agent systems: the inputto-state stability in probability, Automatica, vol. 62, pp. 284-291, 2015.

[3] D. Ding, Z. Wang, J. Lam and B. Shen, Finite-Horizon $H_{\infty}$ control for discrete time-varying systems with randomly occurring nonlinearities and fading measurements, IEEE Transactions on Automatic Control, vol. 60, no. 9, pp. 2488-2493, 2015.

[4] D. Ding, Z. Wang, B. Shen and H. Dong, $H_{\infty}$ state estimation with fading measurements, randomly varying nonlinearities and probabilistic distributed delays, International Journal of Robust and Nonlinear Control, vol. 25, no. 13, pp. 2180-2195, 2015. 
[5] K. Faulstich, R. Gruler, M. Eberhard and K. Haberstroh, Developing rapid mobile POC systems. Part 1:Devices and applications for lateral-flow immunodiagnostics, IVD Technology, vol. 13, no. 6, pp. 47-53, 2007.

[6] P. Hamel and D. Eck, Learning features from music audio with deep belief networks, 11th International Society for Music Information Retrieval Conference, pp. 339-344, 2010.

[7] G. Hinton, Training products of experts by minimizing contrastive divergence, Neural Computation, vol. 14, no. 8, pp. 1771-1800, 2002.

[8] G. Hinton and R. Salakhutdinov, Reducing the dimensionality of data with neural networks, Science, vol. 313, no. 5786, pp. 504-507, 2006.

[9] G. Hinton, L. Deng, D. Yu, G. Dahl, A. Mohamed, N. Jaitly, A. Senior, V. Vanhoucke, P. Nguyen, T. Sainath, and B. Kingsbury, Deep neural networks for acoustic modeling in speech recognition: the shared views of four research groups, IEEE Signal processing magazine, vol. 29, no. 6, pp. 82-97, 2012.

[10] N. Hou, H. Dong, Z. Wang, W. Ren and F. E. Alsaadi, Non-fragile state estimation for discrete Markovian jumping neural networks, Neurocomputing, vol. 179, pp. 238-245, 2016.

[11] L. Huang, Y. Zhang, Ch. Xie, J. Qu, H. Huang, and X. Wang, Research of reflectance photometer based on optical absorption, International Journal for Light and Electron Optics, vol. 121, no. 19, pp. 1725-1728, 2010.

[12] J. Kaur, K. Singh, R. Boro, K. Thampi, M. Raje and G. Varshney, Immunochromatographic dipstick assay format using gold nanoparticles labeled protein-hapten conjugate for the detection of atrazine, Environmental Science and Technology, vol. 41, no. 14, pp. 5028-5036, 2007.

[13] C. Lin, C. Wu, H. Hsu, K. Li and L. Lin, Rapid bio-test strips reader with image processing technology, Optik, vol. 115, no. 8, pp. 363-369, 2004.

[14] D. Li, S. Wei, H. Yang, Y. Li and A. Deng, A sensitive immunochromatographic assay using colloidal gold-antibody probe for rapid detection of pharmaceutical indomethacin in water samples, Biosensors and Bioelectronics, vol. 24, no. 7, pp. $2277-2280,2009$.

[15] Y. Li, N. Zeng and M. Du, A novel image methodology for interpretation of gold immunochromatographic strip, Journal of Computers, vol. 6, no. 3, pp. 540-547, 2011.

[16] J. Li, A. Ouellette, L. Giovangrandi, D. Cooper, A. Ricco and G. Kovacs, Optical scanner for immunoassays with up-converting phosphorescent labels, IEEE Transactions on Biomedical Engineering, vol. 55, no. 5, pp. 1560-1571, 2008.

[17] Y. Liu, F. E. Alsaadi, X. Yin and Y. Wang, Robust $H_{\infty}$ filtering for discrete nonlinear delayed stochastic systems with missing measurements and randomly occurring nonlinearities, International Journal of General Systems, vol. 44, no. 2, pp. 169-181, 2015.

[18] Y. Luo, G. Wei, Y. Liu and X. Ding, Reliable $H_{\infty}$ state estimation for 2-D discrete systems with infinite distributed delays and incomplete observations, International Journal of General Systems, vol. 44, no. 2, pp. 155-168, 2015.

[19] A. Mohamed, T. Sainath, G. Dahl, B. Ramabhadran, G. Hinton, M. Picheny, Deep belief networks using discriminative features for phone recognition, 2011 IEEE International Conference on Acoustics, Speech and Signal Processing, pp. 5060-5063, 2011.

[20] A. Mohamed, G. Dahl, and G. Hinton, Acoustic modeling using deep belief networks, IEEE Transactions on Audio, Speech, and Language Processing, vol. 20, no. 1, pp. 14-22, 2012.

[21] GA. Posthuma-Trumpie, J. Korf and A. van Amerongen, Lateral flow (immuno)assay: its strengths, weaknesses, opportunities and threats. A literature survey, Analytical and Bioanalytical Chemistry, vol. 393, no. 2, pp. 569-582, 2009.

[22] S. Qian and H. Haim, A mathematical model of lateral flow bioreactions applied to sandwich assays, Analytical Biochemistry, vol. 322, no. 1, pp. 89-98, 2003.

[23] S. Qian and H. Haim, Analysis of lateral flow biodetectors: competitive format, Analytical Biochemistry, vol. 326, no. 2, pp. 211-224, 2004.

[24] C. Raphael and Y. Harley, Lateral flow immunoassay, Humana Press, 2008.

[25] E. Sumonphan, S. Auephanwiriyakul and N. Theera-Umpon, Interpretation of nevirapine concentration from immunochromatographic strip test using support vector regression, Proceedings of 2008 IEEE International Conference on Mechatronics and Automation, pp. 633-637, 2008.

[26] R. Srivastava, J. Cheng, D. Wong, and J. Liu, Using deep learning for robustness to parapapillary atrophy in optic disc segmentation, 2015 IEEE 12th International Symposium on Biomedical Imaging, pp. 768-771, 2015.

[27] R. Tanaka, T. Yuhi, N. Nagatani, T. Endo, K. Kerman and Y. Takamura, A novel enhancement assay for immunochromatographic test strips using gold nanoparticles, Anal Bioanal Chem, vol. 385, no. 8, pp. 1414-1420, 2006.

[28] H. Yang, Z. Wang, H. Shu, F. E. Alsaadi and T. Hayat, Almost sure $H_{\infty}$ sliding mode control for nonlinear stochastic systems with Markovian switching and time-delays, Neurocomputing, vol. 175, pp. 392-400, 2016.

[29] Y. Yu, H. Dong, Z. Wang, W. Ren and F. E. Alsaadi, Design of non-fragile state estimators for discrete time-delayed neural networks with parameter uncertainties, Neurocomputing, vol. 182, pp. 18-24, 2016.

[30] H. Wei, Z. Dong, V4 neural network model for shape-based feature extraction and object discrimination, Cognitive Computation, vol. 7 , no. 6, pp. 753-762, 2015.

[31] H. Wei, H. Li, Shape description and recognition method inspired by the primary visual cortex, Cognitive Computation, vol. 6, no. 2, pp. 164-174, 2014.

[32] P. Yager, T. Edwards, E. Fu, K. Helton, K. Nelson, M. R. Tam, B. H. Weigl, Microfuidic diagnostic technologies for global public health, Nature vol. 442, pp. 412-418, 2006.

[33] D. Yu and L. Deng, Deep learning and its applications to signal and information processing, IEEE Signal processing magazine, vol. 28, no. 1, pp. 145-154, 2011. 
[34] H. Yang, Z. Wang, H. Shu, F. E. Alsaadi and T. Hayat, Almost sure $H_{\infty}$ sliding mode control for nonlinear stochastic systems with Markovian switching and time-delays, Neurocomputing, vol. 175, pp. 392-400, 2016.

[35] Y. Yu, H. Dong, Z. Wang, W. Ren and F. E. Alsaadi, Design of non-fragile state estimators for discrete time-delayed neural networks with parameter uncertainties, Neurocomputing, vol. 182, pp. 18-24, 2016.

[36] B. Zineddin, Z. Wang, Y. Shi, Y. Li, M. Du and X. Liu, A multi-view approach to cDNA microarray analysis, International Journal of Computational Biology and Drug Design, vol. 3, no. 2, pp. 91-111, 2010.

[37] N. Zeng, Z. Wang, Y. Li, M. Du and X. Liu, Inference of nonlinear state-space models for sandwich-type lateral flow immunoassay using extended Kalman filtering, IEEE Transactions on Biomedical Engineering, vol. 58, no. 7, pp. 1959-1966, 2011.

[38] N. Zeng, Z. Wang, Y. Li, M. Du and X. Liu, A hybrid EKF and switching PSO algorithm for joint state and parameter estimation of lateral flow immunoassay models, IEEE/ACM Transactions on Computational Biology and Bioinformatics, vol. 9, no. 2, pp. 321-329, 2012.

[39] N. Zeng, Z. Wang, Y. Li, M. Du and X. Liu, Identification of nonlinear lateral flow immunoassay state-space models via particle filter approach, IEEE Transactions on Nanotechnology, vol. 11, no. 2, pp. 321-327, 2012.

[40] N. Zeng, Z. Wang, Y. Li and M. Du, Cellular neural networks for gold immunochromatographic strip image segmentation, Lecture Notes in Computer Science vol. 7231, pp. 110-120, 2012.

[41] N. Zeng, Z. Wang, Y. Li, M. Du, J. Cao and X. Liu, Time series modeling of nano-gold immunochromatographic assay via expectation maximization algorithm. IEEE Trans. Biomedical Engineering, vol. 60, no. 12, pp. 3418-3424, 2013.

[42] N. Zeng, Y.S. Hung, Y. Li, M. Du. A novel switching local evolutionary PSO for quantitative analysis of lateral flow immunoassay, Expert Systems with Application, vol. 41, no. 4, pp. 1708-1715, 2014.

[43] N. Zeng, Z. Wang, B. Zineddin, Y. Li, M. Du, L. Xiao, X. Liu, and T. Young, Image-based quantitative analysis of gold immunochromatographic strip via cellular neural network approach, IEEE Transactions on Medical Imaging, vol. 33, no. 5, pp. 11291136, 2014.

[44] N. Zeng, Z. Wang, H. Zhang, and Fuad E. Alsaadi, A novel switching delayed PSO algorithm for estimating unknown parameters of lateral flow immunoassay, Cognitive Computation, accepted for publication.

[45] J. Zhao, C. Du, H. Sun, X. Liu, J. Sun, Biologically motivated model for outdoor scene classification, Cognitive Computation, vol. 7, no. 1 , pp. 20-33, 2013. 